\section{BMJ Open} Ophthalmology

\title{
Defining the needs and preferences of patients with dry eye disease
}

\author{
Penny Asbell, ${ }^{1}$ Elisabeth Messmer, ${ }^{2}$ Colin Chan, ${ }^{3}$ Gary Johnson, ${ }^{4}$ Brigitte Sloesen, ${ }^{5}$ \\ Nigel Cook ${ }^{5}{ }^{5}$
}

To cite: Asbell P, Messmer E, Chan C, et al. Defining the needs and preferences of patients with dry eye disease. BMJ Open Ophthalmology 2019;4:e000315. doi:10.1136/ bmjophth-2019-000315

- Additional material is published online only. To view please visit the journal online (http://dx.doi.org/10.1136/ bmjophth-2019-000315).

These results have been presented as a poster at the HTAi Congress, Vancouver, 2 June 2018.

Received 16 April 2019

Revised 4 September 2019 Accepted 9 November 2019

D Check for updates

(c) Author(s) (or their employer(s)) 2019. Re-use permitted under CC BY-NC. No commercial re-use. See rights and permissions. Published by BMJ.

${ }^{1}$ Ophthalmology, Icahn School of Medicine at Mount Sinai, New York City, New York, USA ${ }^{2}$ Department of Ophthalmology, Ludwig Maximilians University, Munich, Germany

${ }^{3}$ Central Clinical School, University of Sydney, Sydney Medical School, Sydney, New South Wales, Australia ${ }^{4}$ Inpharmation, High Wycombe, UK

${ }^{5}$ Global Patient Access, Novartis Pharma, Basel, Switzerland

Correspondence to Dr Nigel Cook; nigel.cook@ novartis.com

\section{ABSTRACT \\ Objective Dry eye disease is a multifactorial chronic disease, leading to ocular discomfort and visual disturbance with a substantial impact on quality of life. Therefore, the patient's perspective should be taken into account early in the drug development process. We have developed a step-by-step methodology based on the self-explicated conjoint approach to assess the needs and preferences of patients with moderate-to-severe dry eye disease.}

Methods and Analysis Following a literature review and social media listening (step 0), qualitative phone call interviews were conducted with 12 patients (step 1). Patients' responses underwent content analysis and were coded, quantified and displayed as charts. Based on the emerging trends and attributes identified as relevant in steps 0 and 1 , a quantitative online questionnaire was designed and conducted with 160 patients across four countries (step 2)

Results The online questionnaire was rated as easy/very easy to understand by $60 \%$ of respondents, $62 \%$ rated the survey as easy/very easy to complete and $71 \%$ rated it as interesting/very interesting. Treatment satisfaction was the most important aspect for patients, and the three most relevant attributes were as follows (with the most important indexed to $100 \%$ ): 'treatment effectiveness on symptoms of dry eyes' (100\%), 'frequency of treatment use' $(96 \%)$ and 'how the treatment works' $(95 \%)$.

Conclusion Our methodology was well received by patients, and the results will help inform future clinical trial development and discussions with health technology assessment bodies and regulators on unmet needs and product attributes that are of most value to patients with dry eye disease.

\section{INTRODUCTION}

In clinical practice, treatment outcomes are often dependent on factors relating to patients, including their perception of drug effectiveness, convenience and resulting compliance. ${ }^{12}$ It is therefore important that new treatments address unmet needs from a medical, therapeutic, but also a patient's perspective. Patient input should inform drug design and development early in the process to ensure that endpoints of relevance to patients are included, and hence deliver new products that best address those unmet needs as articulated by the patient community.

\section{Key messages}

What is already known about this subject?

- There is growing evidence that dry eye disease, in particular its more severe form, has a considerable impact on patients' quality of life; however, the medical treatment of this condition remains an unmet need from both the patient's and the physician's perspectives.

\section{What are the new findings?}

The present study reports the design and results of a stepwise methodology based on the self-explicated conjoint approach to assess the needs and preferences of patients with moderate-to-severe dry eye disease.

Following a targeted literature review, social media listening study and telephone interviews, we developed an online quantitative questionnaire, which was administered to 160 patients from 4 countries. Treatment satisfaction was the most important aspect for patients, followed by treatment effectiveness, frequency of use and the therapeutic mode of action.

Most respondents found the questionnaire relevant and easy to understand.

\section{How might these results change research or} clinical practice?

Patient preference studies such as the present one have the potential to inform and guide future drug development strategies and clinical trial design in dry eye disease.

Patient communication and satisfaction in acute illness, and even more so in chronic disease, have thus become a strong focus in the past decade. ${ }^{34}$ Among chronic ocular conditions, dry eye disease (DED) is a multifactorial disease whose understanding, definition and classification have evolved considerably over the past 20 years. ${ }^{5}$ It is now recognised that loss of homeostasis of the tear film is at the centre of DED physiopathology, causing ocular symptoms encompassing discomfort and visual disturbance. DED is accompanied by instability and increased osmolarity of the tear film, which is associated with ocular surface inflammation and damage. Globally, 
DED affects between $5 \%$ and $34 \%$ of the population. Moderate-to-severe DED is associated with significant pain, limitations in performing daily activities, reduced vitality, poor general health, and often depression..$^{5-8}$ The medical treatment of DED, in particular the more severe forms of the disease, remains an unmet need from both a patient and a physician perspective. ${ }^{9} 10$

There is growing evidence that DED has important negative effects on patients' quality of life (QoL). ${ }^{11}$ Disease-specific questionnaires that gather information directly from patients and assess their experiences and needs are therefore essential in the monitoring and management of this chronic ocular condition. ${ }^{12}$

In the present study, we outline a research process and its results in determining the disease symptoms and treatment attributes that are important to patients suffering from moderate-to-severe DED. We have developed a specific step-by-step methodology with a cross-national survey design that has been tested and optimised to define patient needs, preferences and most desirable outcome measures in DED.

This novel approach is intended to inform and guide future drug development strategies and clinical trial design in DED. The results may input into discussions with regulators and health technology assessment (HTA) bodies on product attributes and outcomes that are of most value to patients with DED.

\section{MATERIALS AND METHODS}

\section{Overview of main steps}

Our overall approach is aligned with the good practice recommendations for patient preference research studies. ${ }^{13}$ The methodology used was based on the optimised design of a cross-national survey of preferences of patients living with DED and consisted of a step-by-step research process, as outlined in the following sections. ${ }^{14}$

\section{Desk research: literature review and social media listening (preliminary step 0)}

A targeted literature search of articles published between 2005 and 27 February 2017 on Embase and Medline was conducted to obtain an overview of patients' perspective on DED. The search strategy included both free-text words and medical subject headings and aimed to describe a number of disease-related parameters, such as diagnostic methods, the patient's journey, patient satisfaction with DED and/or treatments, and the unmet needs of patients.

The literature review was followed by an analysis of social media DED content publicly accessible in the English language. ${ }^{15}$ Twitter, blogs/media, forums and newswires (a total of 1192 posts between December 2016 and February 2017) were analysed to source insights on disease burden, diagnosis, treatment patterns and QoL, with data retrieved and collected using a social media data aggregator tool (Social Studio, Salesforce, USA)..$^{15}$

This preliminary research helped us to develop attributes encompassing the full spectrum of needs that were expressed as important to patients and informed the qualitative stage of the project.

The draft survey research questions were designed based on the literature review and the social media listening study with patients. They were then reviewed and critiqued by the authors and discussed with a patient association (the German Association for the Blind and Visually Impaired (DBSV)). The revised research questions were used to finalise the online survey design.

\section{Qualitative telephone interviews (step 1)}

Subsequent indepth telephone interviews (1-1.5 hours each) with patients with moderate and severe DED from the UK, Australia, USA and Germany ( $n=3$ in each country; total $\mathrm{n}=12$ ) were conducted between 26 June and 28 July 2017 to further define the issues and needs of patients in their own words. Inclusion criteria for this step are presented in online supplementary table S1. The questionnaire was translated into German for patients from Germany. The questionnaire was reviewed by the German patient group consulting for this project to ensure that the translated content and wording were fully understandable and correct.

A combination of different qualitative analytical tools was used to analyse patients' responses. Responses were quantified and displayed as charts for multiple-choice questions, whereas for open-ended questions content analysis was first performed to assess emerging patterns. Responses that could not be fit into the predefined themes were used to identify areas for further exploration and were highlighted for inclusion in the subsequent quantitative study.

The qualitative stage was used to validate patient needs found from desk research, test patient understanding of the wording and identify any other patient needs that had not been captured.

\section{Quantitative online questionnaire (step 2)}

The consolidated patient needs that emerged from the research described above were used to develop an online quantitative questionnaire administered to patients with moderate-to-severe DED. The recruitment agency used dry eye patient panels, which included reaching out to patient groups for this indication. The online survey was conducted between 1 November and 20 December 2017.

In addition, we consulted the patient association to ensure that the design of the survey was user-friendly for patients; at the end of the survey, we collected feedback from respondents of their 'survey experience'.

The questionnaire was designed based on a selfexplicated conjoint methodology that quantified patient preferences on a variety of dry eye attributes. ${ }^{16}$

\section{Patients}

A total of 160 patients with moderate-to-severe DED from the UK, Australia, Germany and the USA were recruited (40 patients per country). From previous self-explicated surveys, the typical standardised SD for responses to the 
rating-type questions used is around $20 \%$, translating into an SE of $3 \%$ for a sample size of 40 . It would therefore be unlikely that any practically significant differences in ratings were due to sampling errors.

The study included patients with DED selected by symptoms using standard questionnaires and self-reported diagnosis. Patient selection was conducted through a recruitment screener, which included questions from the Symptoms Bother section of the IDEEL PRO Questionnaire, a validated diagnostic tool used in clinical practice and as part of clinical studies ${ }^{17}$ which allows for the assessment of dry eye severity. The screener also included questions about patients' diagnosis and medication use as prescribed by their eye doctor. A minimum of 15 moderate patients (score 39-65) and 15 severe patients (score $\geq 66$ ) were selected per country.

We also employed a list of screening criteria to ensure that the final sample in each country was representative of the dry eye patient population (online supplementary table S1).

\section{Patient involvement}

A patient support group for the blind in Germany (DBSV) was consulted through regular teleconferences at all stages of the project from design to execution, data analysis and reporting. The preparatory work for this study included a social media listening analysis. The draft survey research questions resulting from the literature review and social media listening study were reviewed and critiqued by three international dry eye clinical experts consulting on this project, as well as by a consultant from DBSV, to ensure that patient perspectives were accurately captured.

Recruitment for this study was carried out using dry eye patient panels. A survey experience assessment was performed at the end of the study to address questions around the time required to complete the survey, ease of completion, ease of understanding and patients' interest in the survey topic.

\section{Self-explicated conjoint}

Conjoint analysis is a well-validated methodology used to elicit preferences for healthcare, ${ }^{18-20}$ based on the premise that respondents' preferences can be calculated based on the value (positive or negative) that they attach to the specific attributes of the disease or product under consideration. It assumes a 'composition rule', stating that every attribute level has some value (or 'utility'), and that the value of the product (or burden of the disease) is equal to the sum of its 'utility part-worths'. ${ }^{21}$

We used the self-explicated conjoint methodology to examine successive ratings of three layers of the surveylevels, attributes and domains-and determine patient utilities. ${ }^{22}$ Within each domain, each attribute was further categorised into multiple levels (online supplementary table S2). To weight the levels within an attribute, respondents were asked to give the best one a score of 100 and rate the others with a relative score between 0 and 100 .
For example, if the attribute 'eye discomfort' was being evaluated, they would consider eye dryness relative to itchy eye relative to eye pressure to assess the extent to which each is responsible for generating 'eye discomfort'.

Respondents then weighted the attributes of each domain relative to each other in the same manner as the levels, that is, by giving the best a score of 100 and scoring the others relative to this; they also weighted the four domains relative to each other in a similar manner. Utilities were calculated by multiplying attribute importance weights by level ratings and are then normalised to sum to $100 \%$.

Conjoint-type methods are the gold standard for patient preference research. Studies have shown that the self-explicated method provides internal and external validity that is at least comparable with other conjointtype preference elicitation techniques. ${ }^{18}{ }^{22-25}$ Indeed, HTA bodies like the National Institute for Health and Care Excellence have recognised the benefits of the self-explicated conjoint method in patient preference research. ${ }^{26}$

The self-explicated approach has equal predictive validity to conventional conjoint methods, is quicker and less expensive to design and analyse, and poses less cognitive burden for patients. ${ }^{27}$ Thus, the self-explicated methodology has the potential to handle multiple attributes and levels with small sample sizes. ${ }^{16}$ Our analysis could thus be performed with tens of respondents, rather than hundreds (which are required for conventional discrete choice experiments). Including multiple attributes is of particular importance in preference studies that are conducted early in the drug development lifecycle, especially in disease areas in which little is known about what features are most relevant to patients. Meanwhile, by comparison with other methods, the selfexplicated approach cannot be used to derive benefit-risk trade-offs, which are important for informing regulatory licensing decisions made much later in the product lifecycle. $^{27}$

\section{RESULTS}

\section{Preliminary steps}

The desk research carried out through a literature review and social media listening allowed us to identify three broad aspects relevant to patients with DED: disease symptoms, impairment of QoL (especially negative impact on daily/work activities and emotions) and patient perceptions of unmet need. ${ }^{15}$

These identified needs and concerns were validated during the interviews, which also improved our understanding of patient perceptions in three ways. First, we uncovered additional needs and concerns including, for example, patients' fear of going blind (online supplementary table S3). Second, we identified areas where our phraseology was unclear to patients; for example, when we mentioned 'irritability', some patients were not sure whether we were talking about eye irritability or emotional irritability. Third, we uncovered examples 
where patients felt that our description of issues involved either duplication or redundancy; for example, the distinction between 'Doing close work in the morning or afternoon' and 'Doing close work in the evening or night' was not considered meaningful.

All these points were addressed in the final survey design by incorporating themes that were found important to patients and by refining the wording of some questions in a patient-friendly way, to optimise the survey experience and outcomes. Example questions are provided as part of the online supplementary information.

\section{Survey design and experience \\ Survey design}

The questionnaire was designed especially for patients with poor eyesight, according to Web Content Accessibility Guidelines. ${ }^{28}$ In particular, it included features such as large font size and good colour contrast. Respondents also had the possibility to stop at any time and return to the survey at a later time or date.

A total of 25 attributes were tested, divided into four domains (table 1): treatment satisfaction, symptom bother, treatment administration and impact on daily life. The levels tested for each attribute are included in online supplementary table S2.

Adverse events were collected and reported as per protocol and a summary report was prepared.

\section{Patient experience}

On average, patients took $50 \mathrm{~min}$ to complete the survey. The patient survey experience was mostly positive, with $71 \%$ of patients finding the survey interesting, $62 \%$ easy to complete and $60 \%$ easy to understand (figure 1 ). The option of allowing patients to stop and return proved to be helpful and was used by a quarter of patients; no participants dropped from the survey without returning.

Fifty-nine per cent of respondents found the length of the survey manageable and $99 \%$ found the survey relevant. Specific examples of feedback are included in online supplementary table $\mathrm{S} 4$.

\section{Main outcomes informing future drug development}

The most important aspects of dry eye to patients as highlighted by the survey results, listed by domains, attributes and levels, are given in figure 2 and table 1. 'Treatment satisfaction' emerged as the most important aspect (domain) to patients with DED (importance of domain $100 \%$, conjoint estimate value 0.253 , $\mathrm{p}<0.05$ vs second most important aspect), followed by 'symptom bother' (importance of domain 90\%, conjoint estimate value $0.227, \mathrm{p}<0.001 \mathrm{vs}$ third most important aspect), 'treatment administration' (importance of domain $72 \%$, conjoint estimate value $0.181, \mathrm{p}=\mathrm{ns}$ vs fourth most important aspect) and 'impact on daily life' (importance of domain $67 \%$, conjoint estimate value 0.169 ; figure 2). The three attributes rated the highest by patients were 'Treatment effectiveness on symptoms of dry eyes' (100\%, conjoint estimate value $0.052, \mathrm{p}<0.05$ difference vs how the treatment works), 'frequency of treatment use' $(96 \%$, conjoint estimate value 0.050 ) and 'how the treatment works' (95\%, conjoint estimate value 0.049 ). The three most bothersome symptoms were 'eye discomfort', 'eye sensitivity' and 'eye pain' (see table 1 for the relative weights of all attributes).

Further insights from this study concerning the differential outcomes of moderate versus severe DED, differences between countries, and results of a QoL assessment will be reported in a separate manuscript. ${ }^{29}$

Based on these results, we were able to gain insights into those dry eye symptoms that bother patients the most and the impact on patients' daily activities, as well as identify unmet needs and salient features with respect to treatment options. Patients expressed a preference for artificial tears administered as drops on an as-needed basis, with a fast onset $(5 \mathrm{~min})$ but also a quick action on the underlying disease. Clinical trials should capture outcomes related to the elimination of eye discomfort, eye pain and eye fatigue, and patients acknowledged the importance of assessing the drug activity on the underlying disease (table 2).

\section{DISCUSSION}

There is an increasing recognition that new treatment options will be more effective and accepted if they address unmet needs and concerns as expressed by the patients. Therefore, patient input is important for designing clinical trials that measure patient-relevant treatment outcomes. In the context of guideline development, it was stated that 'Getting the evidence right-the right options, outcomes, and outcome data-is an obligatory prerequisite for considering informed patient preferences'. ${ }^{30}$ In this study, we developed a stepwise methodology including both a qualitative and a quantitative research approach to determine the relative importance of a wide range of symptoms, their impact on daily activities and potential treatment attributes to patients living with DED.

The quantitative conjoint aspect of our study, based on the self-explicated technique, has proven to be a useful research method and has allowed for a large number of attributes to be researched while using a relatively small sample size ( $\mathrm{n}=160$ across 4 countries). Overall, the survey was very well received and the experience of our participants supports the claim that the self-explicated method is easy to follow without imposing too much cognitive strain on respondents. ${ }^{16}$

Participants found the survey user-friendly, with almost two-thirds rating it as easy or very easy to understand and complete, and over two-thirds rating it as interesting or very interesting. However, one in five patients found the survey challenging and one in four found it too long, thus leaving scope for further improvement in the survey design.

Some of the attributes that emerged from our research were expected and in line with the existing literature. Findings on the impact of DED on daily life (eg, burden 


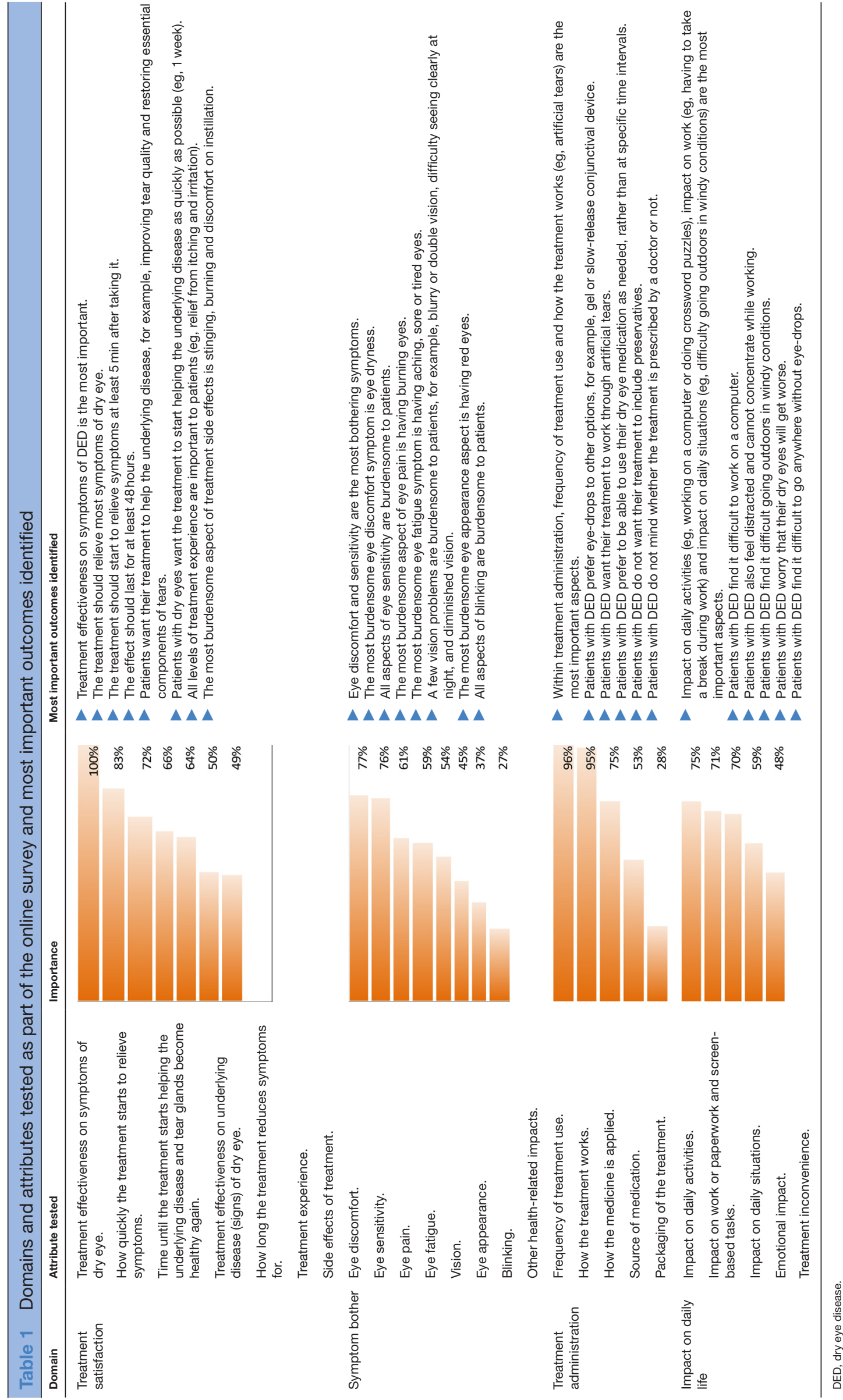


How easy was this survey to understand?

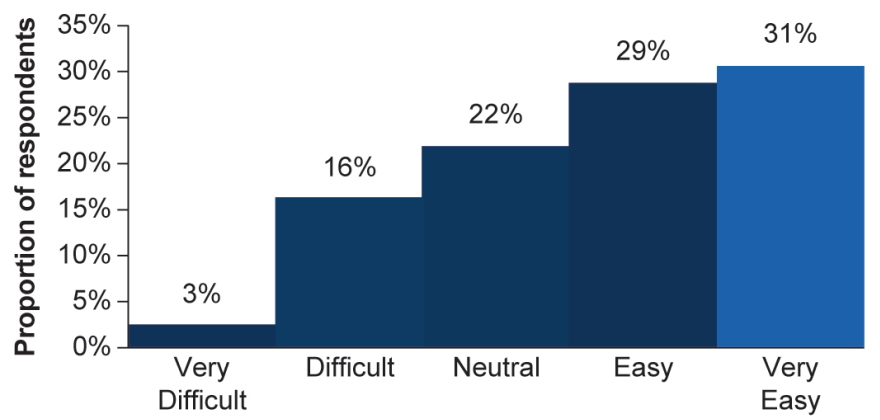

Interest in the questionnaire

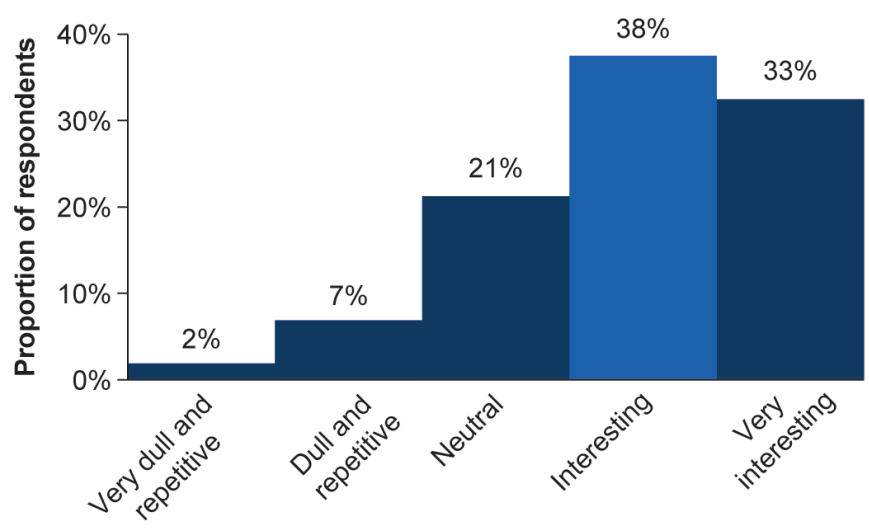

Figure 1 Survey experience.

of working on a computer, feeling distracted and fear of going outdoors in windy conditions) are consistent with a US study on the economic impact (direct/indirect burden) of DED. ${ }^{31}$

Other findings were more surprising or less easy to predict. For instance, although patients suffering from DED do not typically complain about pain during consultation, ocular pain was rated highly in our survey. Patients also worry that their disease may get worse or even lead to blindness (especially for severe DED). By contrast, while most patients reported an impact of DED on their vision, which is to be expected, especially for severe patients, ${ }^{5}$ vision burden did not score very highly in the survey.

Some of the findings highlight a lack of awareness of the existence of an underlying and ongoing disease process, and some patients appear to have contradicting aspirations. On the one hand, patients want their condition to be managed beyond symptom relief. On the other hand, they prefer their treatment to work like artificial tears (for symptom relief only), to act fast (within $5 \mathrm{~min}$ ), be long-lasting ( 48 hours) and to be on an 'as needed' basis.

Fear of dependence may partly explain why patients favour an 'as needed' regimen, which they may associate with more self-control and avoiding overdose. They do not appreciate that in fact the opposite holds true:
How difficult was the survey to complete?

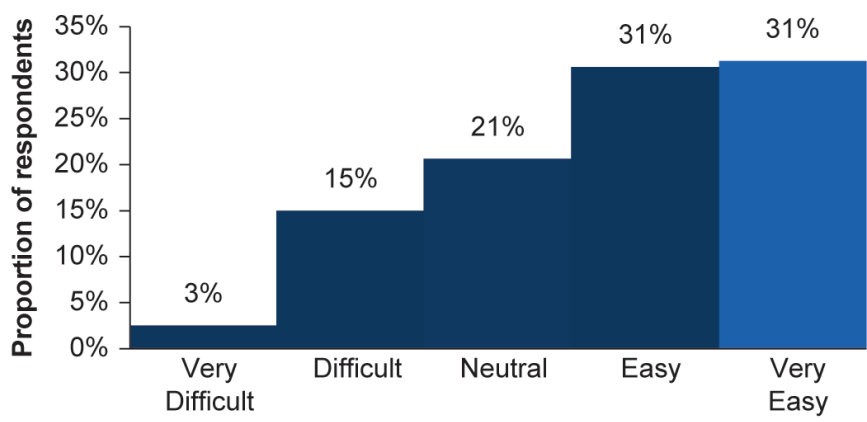

Length of the questionnaire

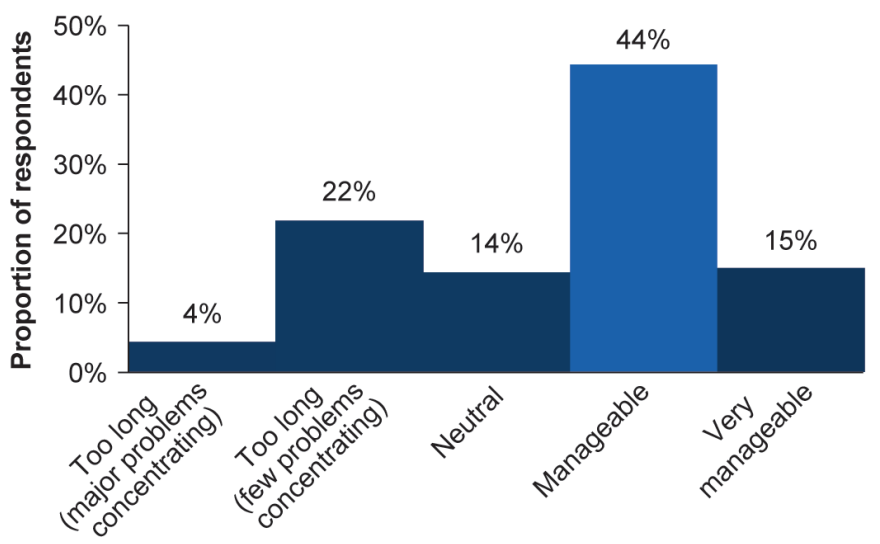

underdosing may result in worsening of the condition, which in turn will increase their reliance on drugs.

The required dosing and expected outcomes are very much dependent on the type of medication. Drops may be in the form of lubricating 'artificial tears' for symptom relief only and on an as-needed regimen, but can also encompass non-steroidal anti-inflammatory drugs, corticosteroid drops, ciclosporin A drops and lifitegrast drops (only available in the USA at the time of the survey), each with different dosing requirements. This may not be clear for patients, who may equate 'drops' with 'artificial

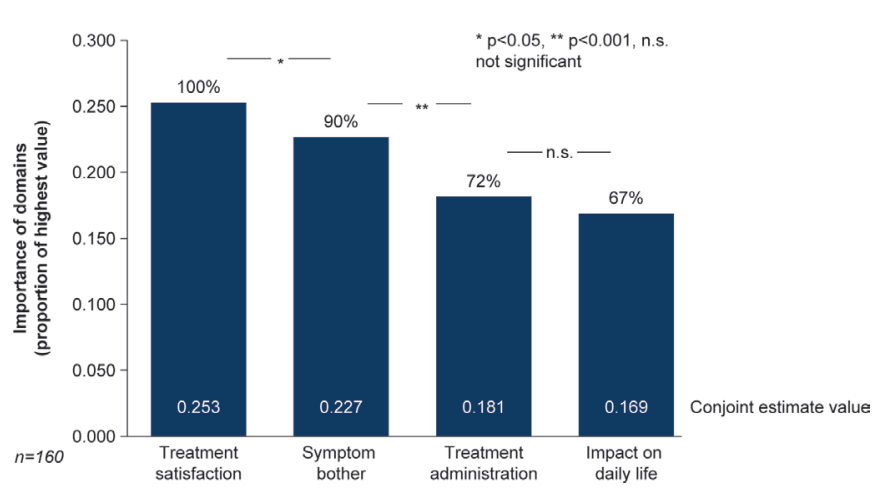

Figure 2 Relative importance of four main aspects (domains). 
Table 2 Optimal product characteristics* and clinical trial outcomes

Optimal product characteristics†

\begin{tabular}{ll} 
Formulation & $\begin{array}{l}\text { Drops (with or without a dropping aid) vs least popular option: slow-release } \\
\text { conjunctival insert. }\end{array}$ \\
\hline Component & Artificial tears (rather than antibacterial or steroids). \\
\hline Dosage & As needed (rather than with specified frequency). \\
Onset of action on symptoms & Within 5 min. \\
\hline Onset of action on underlying disease & As quickly as possible (eg, within a week).
\end{tabular}

Most relevant trial outcomesł

Elimination of dry eyes symptoms

Treatment of the underlying disease

Eye discomfort, including eye dryness (more important than itchy eyes, feeling like something is in your eye, eyes that feel like they have been scratched, crusty or gooey eyes that get stuck shut in the morning, pressure in the eye).

Eye sensitivity, including sensitivity due to light/glare and wind/recirculated air. Eye pain, including general eye pain, burning eyes or stinging eyes. Eye fatigue, including tired eyes and aching or sore eyes.

Repair of eye surface damage. Improvement of tear quality and restoration of the essential components of tears. Reduction in eye redness and inflammation.

${ }^{*}$ Determined by choosing attributes with product characteristics within the 10 most important attributes.

†Some preferences may be related to familiarity, but this underlines areas where a different approach would require some educational effort. ¥Most symptoms (apart from eye pain) can be captured by using the Symptom Bother section of the IDEEL Questionnaire.

tears'; this may have influenced some of the responses in our survey. Good patient communication and education are therefore needed to close the loop between patient expectations and understanding of the disease, its progression and its optimal management. While artificial tears were the preferred treatment option, steroids were not liked by patients, probably because of negative perceptions.

It should be noted that a preference for drops compared with newer options such as implantable slowrelease forms may reflect the fact that the patients tend to 'like what they are already familiar with'. The benefits of inserting any implant in an eye that is already perceived as painful and sensitive may also be counterintuitive. Again, education and encouragement to test out new administration options, supported by clinical trial evidence, may help guide a more informed patient-centred treatment decision and improved disease management.

This study has a number of limitations. One key question is whether the sample we used is representative of the broader DED patient population. Although our sample is to some extent heterogeneous (eg, covering four countries), and controlled by strict inclusion and exclusion criteria, it is still influenced by the methodology used, in that it may preselect patients who are more engaged, active and motivated about managing their condition compared with the overall DED patient population.

Patients' previous treatment experience may also have influenced the results. Preference may vary depending on whether a patient currently uses treatments or not, or whether the patient has experienced a particular symptom or not. Finally, we assume honesty of the respondents since the survey is based on self-completion.
Overall, our study demonstrates that the process of using qualitative research to determine what matters to patients, followed by a quantitative respondent-friendly survey approach, has the potential to identify outcomes that are most relevant to patients. These results should inform future drug development strategies and discussions with regulators, HTAs and payers on patients' unmet needs, preferences and product attributes that are of most value to patients.

Acknowledgements Anna Panpurina (from Inpharmation), Clementine Prince and Sharath Medi (both from Novartis) also provided assistance with the design of the study, and Sandrine Spindler (Novartis) with project management. Marie-Catherine Mousseau, lain Hatch and lleana Stoica (Novartis) provided medical writing support under the guidance of the authors. The authors wish to thank all the survey participants for their involvement in the study.

Contributors All authors contributed to the development of the study concept, and data analysis and interpretation. With regard to the design of this study, the authors were consulted by the Deutscher Blinden und Sehbehindertenverband eV (DBSV) as regulated by a contract between Novartis and DBSV. All authors meet the International Committee of Medical Journal Editors (ICMJE) criteria for authorship for this article and take responsibility for the integrity of the work as a whole. All authors were involved in the preparation and review of the manuscript and approved the final version to be submitted.

Funding The funding/sponsorship of this study and article processing charges were funded by Novartis Pharma, Basel, Switzerland.

Competing interests PA has received research funding from Alcon and MC2 Therapeutics and has participated in the advisory boards of Alcon/Novartis, Allergan and Shire. EM: consultant for Dompe, Pharm Allergan, Santen and Thea, and lecturer for Alcon, Dompe, Pharm Allergan, Santen, Thea and Ursapharm. CC: consultant for Alcon and has participated in the advisory boards of Alcon/ Novartis. NC and BS are employees of Novartis. In addition, NC is a member of IMI PREFER and is also involved with the working groups of ISPOR and HTAi on patient preferences. GJ is an employee of Inpharmation.

Patient consent for publication Obtained.

Ethics approval Our study obtained approval from the Heartland Institutional Review Board, and the research conformed to the codes of both BHBIA (British Healthcare 
Business Intelligence Association) and EphMRA (European Pharmaceutical Market Research Association). These codes of conduct stop this type of research being used as a front for marketing activities and ensure that all adverse event procedures are properly adhered to. Patient cooperation was voluntary, and they were entitled to withdraw at any stage of the process, or subsequently to ask that part or all of the record of their interview was destroyed or deleted. All patients received financial compensation at fair market value in line with the country regulations for their voluntary participation in the survey. The patients were advised that the information they provided would be treated confidentially, without reference to individual names. They were assured that any information they gave would simply be added to the answers received from other respondents to provide an overall picture of views, which may be used in a scientific publication. The online survey was run via a secure website, with data access strictly limited to the patient and the survey support team. Adequate data protection statements were also included.

Provenance and peer review Not commissioned; externally peer reviewed.

Open access This is an open access article distributed in accordance with the Creative Commons Attribution Non Commercial (CC BY-NC 4.0) license, which permits others to distribute, remix, adapt, build upon this work non-commercially, and license their derivative works on different terms, provided the original work is properly cited, appropriate credit is given, any changes made indicated, and the use is non-commercial. See: http://creativecommons.org/licenses/by-nc/4.0/.

ORCID iD

Nigel Cook http://orcid.org/0000-0001-8211-7613

\section{REFERENCES}

1 Cushing A, Metcalfe R. Optimizing medicines management: from compliance to concordance. Therap Clin Risk Manage 2007;3:1047-58.

2 Gerstein BS, Barry MJ, Fowler FJ. How patient centered are medical decisions?: results of a national survey. JAMA Internal Med 2013;173:1215-21.

3 Sleath B, Blalock SJ, Carpenter DM, et al. Ophthalmologist-Patient communication, self-efficacy, and glaucoma medication adherence. Ophthalmology 2015;122:748-54.

4 Thompson AC, Thompson MO, Young DL, et al. Barriers to follow-up and strategies to improve adherence to appointments for care of chronic eye diseases. Invest Ophthalmol Vis Sci 2015;56:4324-31.

5 Craig JP, Nichols KK, Akpek EK, et al. TFOS DEWS II definition and classification report. Ocul Surf 2017;15:276-83.

6 The epidemiology of dry eye disease: report of the epidemiology Subcommittee of the International dry eye workshop (2007). Ocul Surf 2007:5:93-107.

7 The definition and classification of dry eye disease: report of the definition and classification Subcommittee of the International dry eye workshop (2007). Ocul Surf 2007;5:75-92.

8 Bron AJ, Tomlinson A, Foulks GN, et al. Rethinking dry eye disease: a perspective on clinical implications. Ocul Surf 2014;12:S1-31.

9 Messmer EM. The pathophysiology, diagnosis, and treatment of dry eye disease. Dtsch Arztebl Int 2015;112:71-82.

10 Asbell PA, Spiegel S. Ophthalmologist perceptions regarding treatment of moderate-to-severe dry eye: results of a physician survey. Eye Contact Lens 2010;36:33-8.

11 Friedman NJ. Impact of dry eye disease and treatment on quality of life. Curr Opin Ophthalmol 2010;21:310-6.

12 Grubbs JR, Tolleson-Rinehart S, Huynh K, et al. A review of quality of life measures in dry eye questionnaires. Cornea 2014;33:215-8.
13 Berger ML, Sox H, Willke RJ, et al. Good practices for real-world data studies of treatment and/or comparative effectiveness: recommendations from the joint ISPOR-ISPE special Task force on real-world evidence in health care decision making. Value Health 2017;20:1003-8.

14 Kockaya G, Wertheimer A. Pharmaceutical market access in developed markets. SEEd: Torino, 2018.

15 Cook N, Mullins A, Gautam R, et al. Evaluating patient experiences in dry eye disease through social media listening research. Ophthalmology and Therapy. In Press 2019;8:407-20.

16 Sattler H. A comparison of conjoint measurement with selfexplicated approach. In: Gustafsson A, Herrmann A and Huber F (Eds); Conjoint Measurement: Methods and Application [4th edition]. Berlin, Heidelberg: Springer Berlin Heidelberg, 2007. Available: https://epdf.pub/queue/conjoint-measurement-methods-and-appl ications295dc954aeaf13f30e2bbe89a8f992de6211.html [Accessed Aug 2019].

17 Abetz L, Rajagopalan K, Mertzanis P, et al. Development and validation of the impact of dry eye on everyday life (IDEEL) questionnaire, a patient-reported outcomes (pro) measure for the assessment of the burden of dry eye on patients. Health Qual Life Outcomes 2011;9:111.

18 Green PE, Srinivasan V. Conjoint analysis in marketing: new developments with implications for research and practice. J Mark 1990;54:3-19.

19 Ryan M, Farrar S. Using conjoint analysis to elicit preferences for health care. BMJ 2000;320:1530-3.

20 Bridges JF. Stated preference methods in health care evaluation: an emerging methodological paradigm in health economics. Applied Health Econ Health Policy 2003;2:213-24.

21 Green PE. Conjoint measurement for quantifying judgmental data. J Market Res 1971:8:355-63.

22 Srinivasan V, Park CS. Surprising robustness of the Self-Explicated approach to customer preference structure measurement. $J$ Marketing Res 1997;XXXIV:286-91.

23 Green PE, Schaffer CM. Importance Weight Effects on SelfExplicated Preference Models: Some Empirical Findings. In: Holman $\mathrm{RH}$, Solomon MR, eds. Advances in consumer research. Provo, UT: Association for Consumer Research, 1991: 476-82.

24 Agarwal MK, Green PE. Adaptive conjoint analysis versus selfexplicated models: some empirical results. Int J Res Marketing 1991;8:141-6.

25 Sattler H, Hensel-Borner S. A comparison of conjoint measurement with self-explicated approaches. In: Gustafsson A A, Herrmann A Huber F, eds. Conjoint measurement: methods and applications. Heidelberg: Springer-Verlag Berlin, 2001

26 Cowie L, Bouvy J. Measuring patient preferences: an exploratory study to determine how patient preferences data could be used in health technology assessment (HTa), myeloma UK, 2019. Available: https://www.myeloma.org.uk/wp-content/uploads/2019/07/NICEPatient-Preferences-Report.pdf [Accessed Sep 2019].

27 Johnson G. Pharma-Specific conjoint: evidence-based conjoint analysis for an evidence-based industry. Stokenchurch, England: London Scientific Publishing, 2017.

28 Web content accessibility guidelines. Available: https://www.w3.org/ TR/2008/REC-WCAG20-20081211/ [Accessed Jan 2019].

29 Messmer EM. Comparing the needs and preferences of patients with moderate and severe dry eye disease across four countries, 2019.

30 Montori VM, Brito JP, Murad MH. The optimal practice of evidencebased medicine: incorporating patient preferences in practice guidelines. JAMA 2013;310:2503-4.

$31 \mathrm{Yu}$ J, Asche CV, Fairchild CJ. The economic burden of dry eye disease in the United States: a decision tree analysis. Cornea 2011;30:379-87. 